\title{
Reconstruction for massive proximal tibial bone defects using patient-customized three-dimensional-printed metaphyseal cones in revision total knee arthroplasty
}

\section{Yang Li}

Peking University Third Hospital

\section{Xinguang Wang}

Peking University Third Hospital

Hua Tian ( $\square$ tianhua@bjmu.edu.cn )

Peking University Third Hospital https://orcid.org/0000-0001-7139-3372

\section{Research article}

Keywords: Revision total knee arthroplasty, Bone defects, Metaphyseal cone, 3D printing, Patient-customized

Posted Date: June 19th, 2020

DOI: https://doi.org/10.21203/rs.3.rs-36219/v1

License: @ (1) This work is licensed under a Creative Commons Attribution 4.0 International License. Read Full License

Version of Record: A version of this preprint was published at Orthopaedic Surgery on April 25th, 2022. See the published version at https://doi.org/10.1111/os.13282. 


\section{Abstract \\ Background}

The reconstruction of massive bone defects is one of the main challenges in revision total knee arthroplasty (RTKA). Although several methods are available, each of them has its prominent shortcomings. The purpose of this study is to review the clinical outcomes of RTKA with massive proximal tibial bone defects using patientcustomized three-dimensional (3D)-printed highly porous metaphyseal cones.

\section{Methods}

We retrospectively reviewed seven RTKAs with Anderson Orthopaedic Research Institute (AORI) type III tibial defects using patient-customized 3D-printed highly porous metaphyseal cones, which have been performed at a single institution between 2016 and 2018. The mean length of follow-up was 25.3 months (19-36).

\section{Results}

The mean age at diagnosis of the patients was 68 years old (61-77). At the latest follow-up, no aseptic loosening or prosthetic joint infection has been determined. The mean HSS increased from 49 (39-63) to 78 $(70-83)(P<0.01)$; the mean WOMAC increased from $59(46-73)$ to $26(12-38)(P<0.01)$. All patients obtained the range of motion and mechanical alignment improvement postoperatively.

\section{Conclusion}

The patient-customized 3D-printed metaphyseal cone could be a promising technique in addressing severe tibial defects in RTKA. Our study shows encouraging short-term clinical and radiological outcomes with no aseptic loosening, periprosthetic infection, or fracture. Nevertheless, further follow-up and the expansion of sample size are needed to demonstrate the advantage of this innovative technique fully.

\section{Background}

Revision total knee arthroplasty (RTKA) can be challenging by preexisting bone defects caused by periprosthetic infection, aseptic loosening, polyethylene wear, or periprosthetic fractures, which can occur on either the proximal tibia or the distal femur[1]. A full assessment of the features of bone defects, including size, severity, and location, is usually needed preoperatively to determine the most suitable surgical plan. The Anderson Orthopaedic Research Institute (AORI) classification[2] is the most effective and frequently used system for this purpose[3].

AORI type IIB and III defects have been regularly restored with bone graft, or with metal cones or sleeves[4]. These methods increase the difficulty of procedures, the operating time, and the cost[5]. Meanwhile, it is hard to fit the uncustomized cone or sleeve on the defective site, even if additional trimming of the host bone has been performed. The cone-related iatrogenic bone loss would complicate the potential subsequent therapy[6]. Previous researches show the relatively low survival rate of protheses and patient satisfaction in RTKA with massive tibial 
defects. Surgical complications, such as postoperative pain, are common[7, 8]. For all these unsatisfactions, innovative technology is urgently demanded.

The maturity of 3D-printing technology makes the customized cone, modeled following the patient's anatomical construction, a novel solution for bone defect restoration. The cone composed of trabecular titanium imitating natural trabecular bone morphology meets the biomechanical demands, facilitates osseointegration, and induces bone ingrowth $[9,10]$. The high friction from the porous surface also provides splendid immediate fixation. The simplified procedure with shortened operating time is another advantage, especially the massive bone defects are involved[11]. However, the studies focused on the utilization of these patient-customized 3D-printed cones are less reported in RTKA with massive bone defects. The long-term benefits are unclear.

In this study, we present a retrospective review of massive proximal tibial defects RTKAs treated with this novel patient-customized 3D-printed highly porous metaphyseal cones in our institution, which may shed some light on its shot and short-term clinical and radiologic outcomes.

\section{Methods}

\section{Subjects}

This study reviews nine patients with massive proximal tibial defects (AORI type III) who underwent RTKA and received patient-customized 3D-printed highly porous metaphyseal cones in our institution from 2016 to 2018. Two Patients with co-existing massive distal femur defects or incomplete medical records were excluded, leaving seven patients for further evaluation. The mean follow-up was 25.3 months.

\section{Preoperative preparation}

Before establishing surgical procedures, patients received non-contrast CT scans (slice thickness: 0.625) of their affected knees from mid-femur to mid-tibia. Import the CT imaging set (DICOM format) into MIMICS 17 software (Materialise, Belgium) to obtain the 3D model of the proximal tibia. Process the 3D model (STL format) in Unigraphics NX 10.0 software (Siemens PLM Software, Germany). Obtain the appropriate size and model of the tibial prosthesis and diaphyseal stem (ACCK revision knee system, AK Medical, China) by performing virtual bone cutting and prosthesis installation in Unigraphics according to the patient's axial alignment of the lower extremity. Design the suitable cone based on the contour of bone defects and the preselected prosthesis. The upper margin of the cone should be designed to sit at the level of the head of fibula after being appropriately inserted into the host bone, which makes appropriate joint line restoration effortlessly achievable. Export the 3D model of the cone (STL format) into the Electron Beam Melting Rapid Prototyping (EBM-RP) machine (Arcam, Sweden), in which fine titanium powders (Ti6Al4V) are fused into highly porous cone layer-by-layer. The diameter of pores and wires was 600-1000 um and 350-750um, respectively, with an average porosity of 50-80\%. Print another identical polyamide cone as a trial and sterilize with radiation for surgical uses (Fig. 1).

\section{Surgical procedure}

All patient-customized 3D-printed highly porous cones were utilized along with an ACCK revision knee system (AK Medical, China). Sequentially, remove loosening prosthesis or antibiotic-loaded bone cement spacer and all necrotizing soft tissue completely. Then irrigate the articular cavity thoroughly. Double-check the characteristics 
of bone defects. Insert the cone trial. Observe the compatibility of the interface and test for the stability of the insertion. Modify the host bone with high-speed burr or spatula if the initial compatibility was not satisfying. Insert and impact the customized cone into the metaphyseal bone defect appropriately. Implant and cement the tibial plate and stem.

\section{Assessment of outcome}

Patient age, gender, Body Mass Index (BMI), diagnosis, preoperative mechanical axis angle, range of motion(ROM), Hospital for Special Surgery Score (HSS), and Western Ontario and McMaster Universities Osteoarthritis Index (WOMAC) were recorded. Additionally, tibial bone loss AORI classification was determined using preoperative radiographs, as well as intraoperative assessments. Operational characteristics were also recorded, including operating time, total blood loss (TBL), postoperative transfusion, and postoperative complications. The total blood loss is calculated using the Gross equation[12].

Follow-up data, including postoperative mechanical axis angle, HSS, WOMAC, and ROM, were recorded from the latest follow-up. The preoperative and postoperative mechanical axis angle was demonstrated by Hip-Knee-Ankle (HKA) angle.

\section{Statistical analysis}

Statistical significance for preoperative and postoperative HSS and WOMAC was determined using 2-tailed $t$ tests using SPSS 25.0 (SPSS, Inc., Chicago, IL, USA), and P-values less than 0.05 were considered statistically significant.

\section{Results}

The mean age of enrolled patients was 68 years (61-77), with a mean BMI of $28.1(24.9-32.0)$. The primary diagnosis leading to RTKA were aseptic loosening (5/7) and prosthetic joint infection (2/7, Stage II revision). Table 1 displays the demographic features and preoperative characteristics of each patient.

The mean operating time was 108 minutes (95-129). The mean total blood loss (TBL) was $1202 \mathrm{~mL}$ (8901540). Three patients experienced anemia postoperatively and required blood transfusion during hospitalization. Patient 5 had muscular calf vein thrombosis in both legs, which organized after 2-week anticoagulation therapy with low molecular weight heparin (LMWH).

At the latest follow-ups, no aseptic loosening or prosthetic joint infection has been determined. The mean HSS increased from $49(39-63)$ to $78(70-83)(P<0.01)$; the mean WOMAC increased from $59(46-73)$ to 26 (12-38) $(P<0.01)$. There was no trace of progressive radiolucent lines or radiographic loosening. The mean flexion angle increased to $93^{\circ}(80-100)$ from $66^{\circ}(30-80)$. Meanwhile, the mechanical axial has been well restored, with all the HKA angles within $\pm 3^{\circ}$. Table 2 lists the operational characteristics and follow-up data. Figure 2 displays the perioperative radiographs for Patient 1.

\section{Discussion}

The volume of RTKA is rising as the aging of the population and the consequently increasing demand for primary TKA. 268,000 RTKAs are expected in the United States by 2030, six times the number in 2005[13]. The restoration of the proximal tibial defect is one of the main challenges in RTKA. The reconstructing strategy usually varies 
according to the extent of bone defects. The Anderson Orthopedic Research Institute (AORI) classification is the most practical and widely accepted system among them for preoperative planning.

For mild proximal tibial defects (AORI type I and IIA), good clinical outcomes have been achieved by applying bone cement, metal screw, bone grafting, or metal augment[14]. On the other hand, for more severe bone defects, especially AORI type III, each traditional restoration technique has its own prominent shortcomings.

The bone grafting technique is well adopted for massive bone defects reconstruction, including impaction bone grafting and structural bone grafting. It allows surgeons to restore various bone defects in different shapes and sizes and maintain the joint line. Compared with the expansive metal augment, bone grafting is a more economical solution, especially when autologous grafts are feasible. However, prosthesis collapse as a typical result of non-fusion grafting would be catastrophic. The study reported by Hilgen et al.[15] shows that the 10-year survival of the prosthesis was only $50 \%$, in which bone grafting was used for tibial AORI type II and type III defects. In another study, Engh et al.[16] enrolled 46 RTKA patients with proximal tibial AORI type III defects who underwent structural bone grafting and followed-up for an average of 97 months. Although the 10-year survival of the prosthesis reaches $90 \%$, four prosthesis failures occurred which caused by infection, aseptic loosening, osteonecrosis, or polyethylene wear. Besides, the bone grafting utilization has been further limited by the availability of allograft, the complexity of grafting procedure, and the requirement of experienced surgeons[17].

Recently, cones and sleeves are becoming the mainstream of tibial metaphyseal bone defects restoration. Cones have better performance in filling defects, while sleeves focus more on stability. The multiplicity of their size and shape allows them to fit various bone defects. High friction introduced by the porous metal surface facilitates immediate metaphyseal stability, which makes postoperative early weight-bearing probable. Meanwhile, the bone-ingrowth-friendly porous structure will lead to excellent long-term fixation and clinical outcomes. A study of 66 proximal tibial defects (AORI type IIB or III) restoration with tantalum cones in RTKA conducted by Kamath et al.[18] shows $95 \%$ survival of the proximal tibial prosthesis on an average of 70-month follow-up. In another similar retrospective study reported by Burastero et al.[19] on an average of 43-month follow-up, the cone-related survival rate was $97.8 \%$ of 60 cases of RTKA with massive bone defects. It also shows significant improvement in patients' postoperative joint functions and knees ROM. Klim et al.[20] conducted a 6.3-year follow-up of 93 RTKA with bone defects restored with metaphyseal sleeves. Not even a single case shows aseptic loosening. Even though 17 cases underwent reoperation due to recurrent infection or periprosthetic fracture, 96.1\% (73/76) of the rest cohort achieved excellent radiographic osseointegration with significant improvement of functions and ROM of the knee. Zanirato et al.[21] systemically reviewed 37 published studies of RTKA with bone defects restoration, 927 cone cases (3.6-year average follow-up), and 1801 sleeve cases (4.5-year average follow-up) were enrolled. Both cone and sleeve groups showed promising clinical and functional outcomes. The aseptic survivorship of the implants was $97.3 \%$ in cones groups and $97.8 \%$ in sleeves group.

Although metaphyseal cones and sleeves have achieved good survivorship and clinical outcomes, some drawbacks have emerged. Precise anatomic reconstruction and biomechanic restoration are challenging to accomplish in that uncustomized cones and sleeves cannot perfectly fit into various bone defects. During the cone or sleeve insertion, further sculpturing and reaming of the host bone with a broach or high-speed burr are always required to optimize the bone-implant contact and enhance subsequent bone ingrowth. The additional bone sculpturing would multiples the procedure complexity, prolongs the operating time and introduces 
iatrogenic bone loss. The intraoperative fracture caused by implants insertion is another unneglectable risk, which increases the difficulty of the procedure even more[22].

Given the advancement of 3D printing technology, 3D-printed implants are also available in the market for bone defect reconstruction in RTKA. A study of 62 RTKA cases, in which metaphyseal tibial cones were applied for AORI type II or III tibial defects, followed-up on average of 27 months by Denehy et al.[23] shows that the revisionfree survival of the cones was $90.2 \%$ (the survivorship was $100 \%$ if the infection was excluded) with the notable improvement of patients' postoperative functions. Even though the 3D-printed cones were involved in this study, they were manufactured into several fixed sizes (symmetrical and unsymmetrical). Intraoperative bone sculpturing is inevitable to attain an ideal anatomical fit between the uncustomized cone and various bone defects. 24\%(15/60) cases among them required additional operations. Yin et al.[24] reported the application of individually customized 3D-printed porous titanium augment in a single RTKA case to reconstruct the proximal tibial defect (AORI type IIB), which was caused by previous periprosthetic infection. The prosthesis stability and the clinical outcome were satisfying at the 6-month follow-up. However, the porous alloy was only used as a filler to restore the bone defect in this study. Metaphyseal fixation was not obtained. Furthermore, the bone defect was relatively shallow, although it was classified as AORI type IIB. The short follow-up period is another limitation.

All the cones utilized in our study for RTKA with AORI type III tibial defects were modeled based on the patients' preoperative CT scans and printed layer-by-layer with titanium alloy. From our experience, immediate rigid metaphyseal fixation was much more accessible to achieve with the customized cones compared with fixed-size ones. Both operating time and procedure complexity were decreased. On the 25-month follow-up, clinical outcomes were excellent, without complications such as aseptic loosening, periprosthetic infection, and fracture.

Our study reveals several advantages of the application of 3D-printed patient-customized cones in RTKA with massive proximal tibial defects. High stability stands out firstly. By combining the excellent filling performance of conventional cones with the prominent stability of sleeves, customized cones can achieve reliable initial metaphyseal fixation. The pore size and porosity of the cones can be appropriately configured in modeling to increase the friction and promote the osseointegration, which respectively maximizes the initial stability and long-term stability[25]. Thanks to their incredible immediate stability, the demand for diaphyseal stems fixation could be minimized or even eliminated to avoid the stem-related postoperative leg pain. Further searches, including finite element analysis, are required to verify this benefit, which is our next step.

Excellent fitness, both functionally and anatomically, is another main advantage. The 3D-printed patientcustomized cone modeled according to the preoperative images fits the bone defects much more precisely compared to the conventional fixed-size cones, especially when dealing with massive bone defects in complicated revisions. Since it is an anatomical copy of the patient's knee, a customized cone can be implanted with minimal postoperative soft tissue interference. It obtains both bone and soft tissue fit.

Besides, As the extra bone cutting and reaming minimized by the utilization of customized cones, not only the iatrogenic bone loss is significantly avoided, but also the procedure greatly simplified by converting a complicated revision TKA into a straightforward "primary" TKA. Metal 3D printing technology also immensely reduces the cost of the customized cone and shortens its production cycle since traditional manufacturing techniques, such as casting, forging, and cutting, are no longer involved, making it a more practical and affordable solution[26]. Moreover, the 3D-printed porous cone can accurately mimic the elastic modulus gradient from cancellous bone to cortical bone in order to diminish stress shielding and osteolysis[27]. 
There are some limitations to our study. We enrolled in seven cases without a control group; nevertheless, it is by far the largest case series regarding the utilization of the patient-customized 3D-printed metaphyseal cone in RTKA with massive bone defects. The length of the follow-up period is still short. Thus the comparison with longterm clinical and radiological outcomes of traditional cones in RTKA is unavailable. Coincidentally, all seven cases are female patients, which may induce selection bias to some extent. In order to further verify the advantages of this technique, the expansion of the sample size and follow-up time is needed for subsequent studies.

\section{Conclusion}

The patient-customized 3D-printed highly porous metaphyseal cone could be a promising technique in addressing severe tibial defects (AORI type III) in RTKA, as it effectively simplifies the procedures, shortens the operating time, and achieves articular biomechanical stability. Our study shows encouraging short-term clinical and radiological outcomes with no aseptic loosening, periprosthetic infection, or fracture. Nevertheless, further follow-up and the expansion of sample size are needed to demonstrate the advantage of this innovative technique fully.

\section{Abbreviations}

RTKA: revision total knee arthroplasty; AORI: Anderson Orthopaedic Research Institute; CT: computed tomography; EBM-RP: Electron Beam Melting Rapid Prototyping; BMI: Body Mass Index; ROM: range of motion; HSS: Hospital for Special Surgery Score; WOMAC: Western Ontario and McMaster Universities Osteoarthritis Index; TBL: total blood loss; HKA: Hip-Knee-Ankle; LMWH: low molecular weight heparin.

\section{Declarations}

\section{Acknowledgements}

We appreciate the contribution of all patients and the medical staff.

\section{Authors' contributions}

YL and XW are joint first authors. YL and HT designed this study. $Y L, X W$ and HT were involved in surgical procedure, data collecting and statistical analysis. YL and XW drafted the manuscript. HT contributed to critical revision of the manuscript. HT was the study guarantor. All authors have read and approved the final manuscript.

\section{Funding}

None.

\section{Availability of data and materials}

The data analyzed during the current study are always available from the corresponding author on reasonable request.

\section{Ethics approval and consent to participate}


This study was approved by the Ethics Committee of Peking University Third Hospital. All patients signed informed consent.

\section{Consent for publication}

All patients signed informed consent.

\section{Competing interests}

All authors declare that they have no competing interests.

\section{References}

1. Ponzio DY, Austin MS. Metaphyseal bone loss in revision knee arthroplasty. Curr Rev Musculoskelet Med. 2015;8(4):361-7.

2. Engh GA, Parks NL. The management of bone defects in revision total knee arthroplasty. Instr Course Lect. 1997;46:227-36.

3. Shafaghi R, Rodriguez O, Schemitsch EH, Zalzal P, Waldman SD, Papini M, Towler MR. A review of materials for managing bone loss in revision total knee arthroplasty. Mater Sci Eng C Mater Biol Appl. 2019;104:109941.

4. Sheth NP, Bonadio MB, Demange MK. Bone Loss in Revision Total Knee Arthroplasty: Evaluation and Management. J Am Acad Orthop Surg. 2017;25(5):348-57.

5. Sculco PK, Abdel MP, Hanssen AD, Lewallen DG. The management of bone loss in revision total knee arthroplasty: rebuild, reinforce, and augment. Bone Joint J. 2016;98-B(1 Suppl A):120-124.

6. You JS, Wright AR, Hasegawa I, Kobayashi B, Kawahara M, Wang J, Nakasone CK. Addressing large tibial osseous defects in primary total knee arthroplasty using porous tantalum cones. Knee. 2019;26(1):228-39.

7. Abdelaziz H, Jaramillo R, Gehrke T, Ohlmeier M, Citak M. Clinical Survivorship of Aseptic Revision Total Knee Arthroplasty Using Hinged Knees and Tantalum Cones at Minimum 10-Year Follow-Up. J Arthroplasty. 2019;34(12):3018-22.

8. Petersen KK, Simonsen O, Laursen MB, Nielsen TA, Rasmussen S, Arendt-Nielsen L. Chronic postoperative pain after primary and revision total knee arthroplasty. Clin J Pain. 2015;31(1):1-6.

9. Faizan A, Bhowmik-Stoker M, Alipit V, Kirk AE, Krebs VE, Harwin SF, Meneghini RM. Development and Verification of Novel Porous Titanium Metaphyseal Cones for Revision Total Knee Arthroplasty. J Arthroplasty. 2017;32(6):1946-53.

10. Cheng A, Humayun A, Cohen DJ, Boyan BD, Schwartz Z. Additively manufactured 3D porous Ti-6Al-4V constructs mimic trabecular bone structure and regulate osteoblast proliferation, differentiation and local factor production in a porosity and surface roughness dependent manner. Biofabrication. 2014;6(4):045007.

11. Brune JC, Hesselbarth U, Seifert P, Nowack D, von Versen R, Smith MD, Seifert D. CT Lesion Model-Based Structural Allografts: Custom Fabrication and Clinical Experience. Transfus Med Hemother. 2012;39(6):395404.

12. Gross JB. Estimating allowable blood loss: corrected for dilution. Anesthesiology. 1983;58(3):277-80. 
13. Kurtz S, Ong K, Lau E, Mowat F, Halpern M. Projections of primary and revision hip and knee arthroplasty in the United States from 2005 to 2030. J Bone Joint Surg Am. 2007;89(4):780-5.

14. Lei PF, Hu RY, Hu YH. Bone Defects in Revision Total Knee Arthroplasty and Management. Orthop Surg. 2019;11(1):15-24.

15. Hilgen V, Citak M, Vettorazzi E, Haasper C, Day K, Amling M, Gehrke T, Gebauer M. 10-year results following impaction bone grafting of major bone defects in 29 rotational and hinged knee revision arthroplasties: a follow-up of a previous report. Acta Orthop. 2013;84(4):387-91.

16. Engh GA, Ammeen DJ. Use of structural allograft in revision total knee arthroplasty in knees with severe tibial bone loss. J Bone Joint Surg Am. 2007;89(12):2640-7.

17. Richards CJ, Garbuz DS, Pugh L, Masri BA. Revision total knee arthroplasty: clinical outcome comparison with and without the use of femoral head structural allograft. J Arthroplasty. 2011;26(8):1299-304.

18. Kamath AF, Lewallen DG, Hanssen AD. Porous tantalum metaphyseal cones for severe tibial bone loss in revision knee arthroplasty: a five to nine-year follow-up. J Bone Joint Surg Am. 2015;97(3):216-23.

19. Burastero G, Cavagnaro L, Chiarlone F, Alessio-Mazzola M, Carrega G, Felli L. The Use of Tantalum Metaphyseal Cones for the Management of Severe Bone Defects in Septic Knee Revision. J Arthroplasty. 2018;33(12):3739-45.

20. Klim SM, Amerstorfer F, Bernhardt GA, Sadoghi P, Hauer G, Leitner L, Leithner A, Glehr M. Excellent mid-term osseointegration and implant survival using metaphyseal sleeves in revision total knee arthroplasty. Knee Surg Sports Traumatol Arthrosc. 2020.[published online ahead of print, 2020 Jan 31].

21. Zanirato A, Formica M, Cavagnaro L, Divano S, Burastero G, Felli L. Metaphyseal cones and sleeves in revision total knee arthroplasty: Two sides of the same coin? Complications, clinical and radiological resultsa systematic review of the literature. Musculoskelet Surg. 2020;104(1):25-35.

22. Barnett SL, Mayer RR, Gondusky JS, Choi L, Patel JJ, Gorab RS. Use of stepped porous titanium metaphyseal sleeves for tibial defects in revision total knee arthroplasty: short term results. J Arthroplasty. 2014;29(6):1219-24.

23. Denehy KM, Abhari S, Krebs VE, Higuera-Rueda CA, Samuel LT, Sultan AA, Mont MA, Malkani AL. Metaphyseal Fixation Using Highly Porous Cones in Revision Total Knee Arthroplasty: Minimum Two Year Follow Up Study. J Arthroplasty. 2019;34(10):2439-43.

24. Yin Q, Liu W, Wang S. Application of customized augments fabricated by rapid prototyping for severe bone defects of the knee. Chin Med J (Engl). 2014;127(15):2870-1.

25. Karageorgiou V, Kaplan D. Porosity of 3D biomaterial scaffolds and osteogenesis. Biomaterials. 2005;26(27):5474-91.

26. Malik HH, Darwood AR, Shaunak S, Kulatilake P, El-Hilly AA, Mulki O, Baskaradas A. Three-dimensional printing in surgery: a review of current surgical applications. J Surg Res. 2015;199(2):512-22.

27. Parthasarathy J, Starly B, Raman S, Christensen A. Mechanical evaluation of porous titanium (Ti6Al4V) structures with electron beam melting (EBM). J Mech Behav Biomed Mater. 2010;3(3):249-59.

\section{Tables}

Table 1: Demographics and Preoperative Characteristics of Patients 


\begin{tabular}{|c|c|c|c|c|c|c|c|c|}
\hline Paitent \# & $\begin{array}{c}\text { Age at } \\
\text { Diagnosis(yrs) }\end{array}$ & Gender & $\begin{array}{c}\mathrm{BMI} \\
\left(\mathrm{kg} / \mathrm{m}^{2}\right)\end{array}$ & Diagnosis & $\begin{array}{c}\text { Preoperative } \\
\mathrm{ROM}\left({ }^{\circ}\right)\end{array}$ & $\begin{array}{c}\text { Preoperative } \\
\text { HSS }\end{array}$ & $\begin{array}{c}\text { Preoperative } \\
\text { WOMAC }\end{array}$ & $\begin{array}{l}\text { Preoperative } \\
\text { HKA angle }\left({ }^{\circ}\right)\end{array}$ \\
\hline 1 & 65 & $\mathrm{~F}$ & 30.84 & prosthetic joint infection & $10-80$ & 39 & 73 & +7.2 \\
\hline 2 & 61 & $\mathrm{~F}$ & 32.04 & prosthetic joint infection & $0-30$ & 44 & 66 & +8.1 \\
\hline 3 & 67 & $\mathrm{~F}$ & 27.24 & aseptic loosening & $10-70$ & 51 & 51 & +19.7 \\
\hline 4 & 69 & $\mathrm{~F}$ & 26.18 & aseptic loosening & $15-80$ & 54 & 62 & +20.6 \\
\hline 5 & 77 & $\mathrm{~F}$ & 28.40 & aseptic loosening & $0-80$ & 63 & 46 & +10.8 \\
\hline 6 & 73 & $\mathrm{~F}$ & 24.97 & aseptic loosening & $5-60$ & 42 & 59 & +18.5 \\
\hline 7 & 64 & $\mathrm{~F}$ & 27.06 & aseptic loosening & $10-65$ & 48 & 55 & +7.6 \\
\hline
\end{tabular}

Abbreviations: \#: number; yrs: years; F: Female; +: Varus.

Table 2: Surgical Characteristics and Follow-up Data

\begin{tabular}{|c|c|c|c|c|c|c|c|c|c|}
\hline $\begin{array}{c}\text { Paitent } \\
\#\end{array}$ & operatingtime(min) & $\begin{array}{c}\text { Estimated } \\
\text { Blood } \\
\text { Loss(mL) }\end{array}$ & $\begin{array}{c}\text { Postoperative } \\
\text { Transfusion }\end{array}$ & $\begin{array}{l}\text { Postoperative } \\
\text { complications }\end{array}$ & $\begin{array}{l}\text { Follow- } \\
\text { up } \\
\text { (months) }\end{array}$ & $\begin{array}{c}\text { Postoperative } \\
\operatorname{ROM}\left({ }^{\circ}\right)\end{array}$ & $\begin{array}{c}\text { Postoperative } \\
\text { HSS }\end{array}$ & $\begin{array}{c}\text { Postoperative } \\
\text { WOMAC }\end{array}$ & $\begin{array}{l}\text { Postoperative } \\
\text { HKA angle( }\left(^{\circ}\right)\end{array}$ \\
\hline 1 & 129 & 890.6 & No & No & 36 & $0-100$ & 83 & 12 & -1.1 \\
\hline 2 & 102 & 1445.9 & No & No & 27 & $0-90$ & 70 & 38 & +1.8 \\
\hline 3 & 95 & 1540.2 & Yes, $400 \mathrm{~mL}$ & No & 19 & 0-95 & 80 & 35 & +2.9 \\
\hline 4 & 112 & 1064.2 & Yes, $200 \mathrm{~mL}$ & No & 24 & $0-95$ & 82 & 27 & +1.3 \\
\hline 5 & 103 & 1140.3 & No & $\begin{array}{c}\text { Calf } \\
\text { thrombosis }\end{array}$ & 21 & $5-95$ & 78 & 16 & -2.3 \\
\hline 6 & 115 & 1383.2 & Yes, $400 \mathrm{~mL}$ & No & 24 & $0-80$ & 74 & 26 & 0.3 \\
\hline 7 & 99 & 949.8 & No & No & 26 & $10-95$ & 77 & 29 & -1.9 \\
\hline
\end{tabular}

Abbreviations: \#: number; min: minutes; +: Varus; -: Valgus.

Figures 


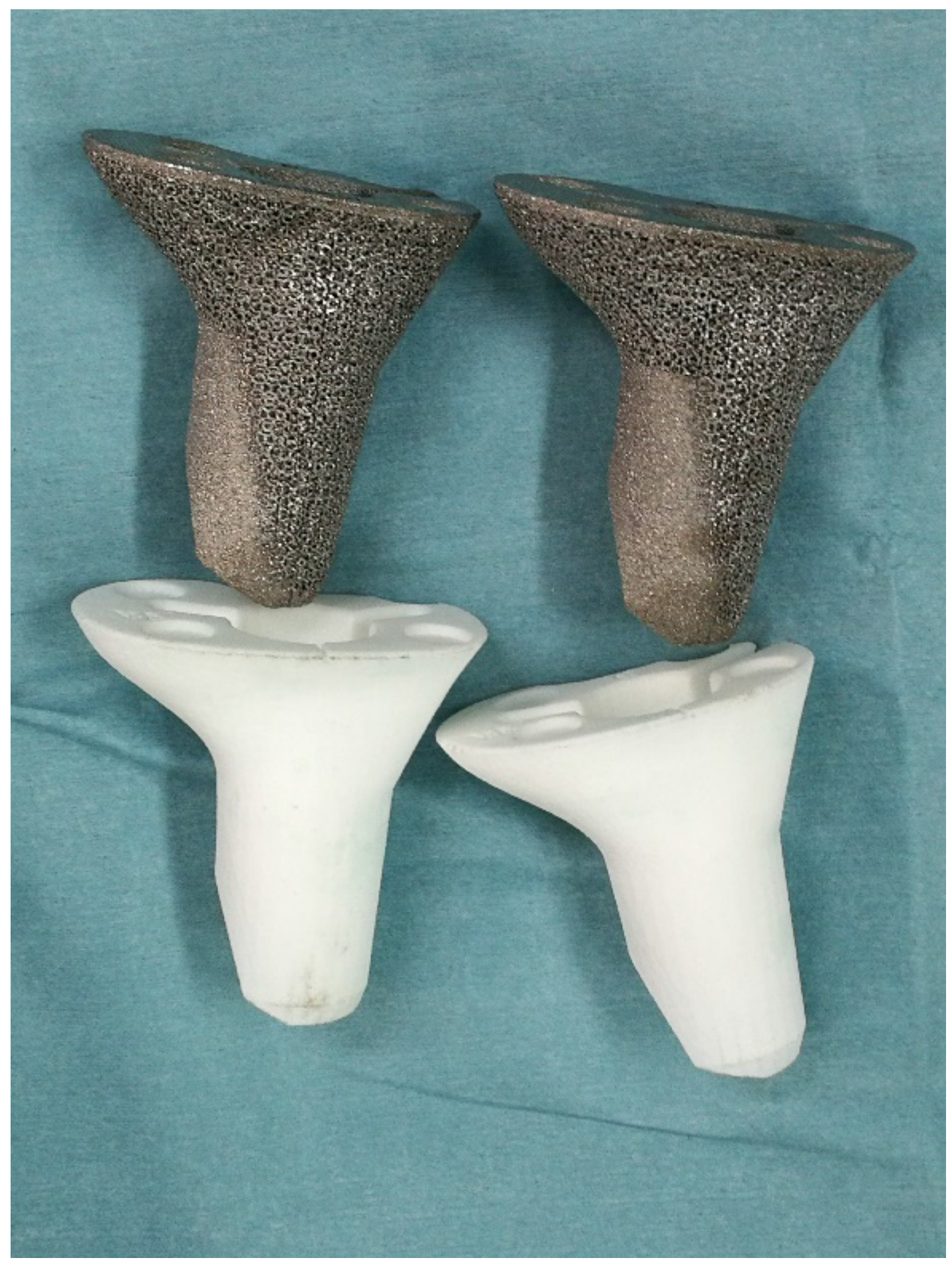

\section{Figure 1}

patient-customized 3D-printed highly porous metaphyseal cones and their trials (This photo was taken by Dr. Yang Li during the operation and we have the copyright of this image). 

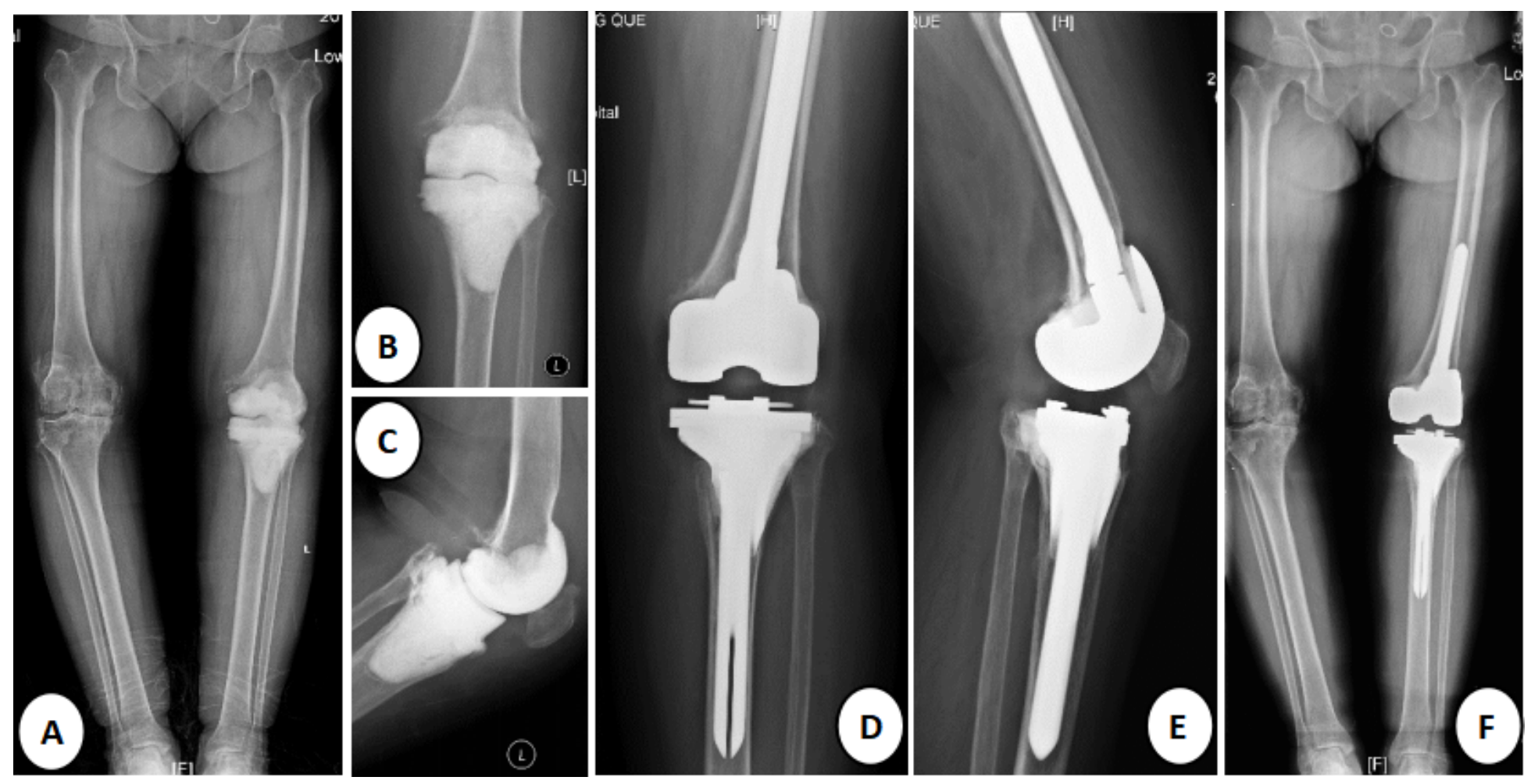

Figure 2

Representative images for patient 1. (A, B, C) Preoperative AP/lateral X-rays which show antibiotic-loaded cement spacers. The patient had been debrided due to the periprosthetic infection. (D, E, F) AP/lateral X-rays at the latest follow-up (36 months). 